\title{
LA AGENDA 2030 EN IBEROAMÉRICA: VISIÓn Y MISIÓn DESDE EL ÁMBITO LOCAL
}

\author{
The 2030 Agenda in Iberoamerica: Vision and \\ Mission from the Local Level
}

\author{
Juana López Pagan \\ Universidad Complutense de Madrid \\ E-mail: juanalop@ucm.es
}

0 Autor

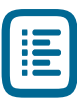

Resumen diseño de la nueva Agenda 2030 de Desarrollo Sostenible y de la Nueva Agenda Urbana (NAU). Ambas agendas muestran la importancia que ostentan los Gobiernos locales y regionales para garantizar una implementación efectiva de los objetivos propuestos. El caso de Iberoamérica es especialmente relevante. La consecución de dichas agendas internacionales necesita de un nuevo enfoque de colaboración de gobernanza abierta multinivel, en el cual el diálogo y la complementariedad de los diferentes niveles de Gobierno (central, regional y local) sea constante y constructivo y donde las interacciones entre el sector público, el sector privado, la academia, la sociedad civil y la propia ciudadanía sean regulares y fluidas. En este sentido, tres de los ODS son considerados particularmente relevantes: el ODS 11, que hace referencia a las ciudades sostenibles, el ODS 16, que aboga por la creación de instituciones eficaces, responsables e inclusivas y el ODS 17, que llama a la creación y revitalización de alianzas entre los mencionados actores para alcanzar los objetivos de manera eficaz.

Agenda 2030; gobernanza multinivel; transparencia; participación; gobierno abierto. 2030 Agenda; multilevel governance; transparency; participation; open government.

Key uords 
Local governments and the associations representing them have actively participated in the design of the 2030 Development Agenda and the Sustainable Development Goals (SDG) and have actively contributed to the negotiations of the New Urban Agenda (NUA). Both agendas show the importance of the role that cities and territorial governments of all sizes have to ensure effective implementation of the proposed objectives. The case of Iberoamerica is especially relevant. The achievement of these international agendas requires a new approach of multilevel open governance, where the dialogue and complementary actions between different levels of government (central, regional and local) are constant and constructive and where the interactions between the public, private sector and civil society are easy and regular. In this regard, three of the SDGs are considered particularly relevant: SDG 11, which refers to sustainable cities, SDG 16, which calls for the creation of effective, responsible and inclusive institutions and SDG 17, which appeals to the creation and revitalization of alliances between the aforementioned actors in order to achieve the objectives effectively.

\section{La Agenda 2030: de la aprobación a la localización}

La Agenda 2030 de los objetivos de desarrollo sostenible (ODS) constituye la primera agenda global y omnicomprensiva aprobada por el conjunto de la comunidad internacional, resultado de los esfuerzos realizados a partir de la Declaración del Milenio del año 2000, en el seno de la Asamblea General de Naciones Unidas. La Agenda de Desarrollo Sostenible materializa, así, los debates y esfuerzos desarrollados por la comunidad internacional en pro del desarrollo humano sostenible desde sus múltiples dimensiones: el desarrollo de las personas, el crecimiento económico, la sostenibilidad ambiental, las instituciones democráticas y justas, y la conformación de una alianza internacional para su consecución (FEMP, 2018a). A pesar de los logros obtenidos en el marco de la Agenda de los Objetivos de Desarrollo del Milenio (ODM), es imprescindible señalar que aún persisten enormes desafíos. De ahí que, los ODS hayan surgido con la intención de fomentar un modelo de desarrollo que aborde las reformas necesarias para hacer frente a estos retos y a otros muchos, poniendo en el centro de los programas de acción a las personas, bajo el principio de "no dejar a nadie atrás” (FEMP, 2018a).

Sin embargo, el potencial de los ODS solo podrá realizarse de manera plena, en tanto que se diseñen instituciones y estructuras estables capaces de garantizar su desarrollo. Ello, supone la apuesta por un modelo de gobernanza que incorpore y revalorice alianzas de diferentes actores en el territorio para promover un enriquecimiento mutuo y una adecuada generación de sinergias tal y como promueve el ODS 17 "Alianzas para lograr los objetivos". La Agenda de Desarrollo Sostenible "es más compleja y exhaustiva que los anteriores programas de actuación aprobados por Naciones Unidas, incluye más dimensiones físicas y ambientales, y debe, además, buscar coordinación con otros compromisos internacionales como el Acuerdo de París sobre Cambio Climático o la Nueva Agenda Urbana, con los que se complementa. Por ello, hoy nos enfrentamos a una serie de importantes retos para la correcta implementación de la agenda, entre los que cabe destacar los siguientes" (FEMP, 2018a):

» Los ODS deben ubicar a las personas en el centro del desarrollo económico contribuyendo a fomentar un modelo de crecimiento más equilibrado y sostenible, con mejor base social, evitando nuevas desigualdades y riesgos. Para ello, es imprescindible que la Agenda de Desarrollo Sostenible no se sitúe en la periferia de la formulación de las políticas económicas. 
» La Agenda 2030 es resultado de la nueva relación existente entre países industrializados, emergentes y en desarrollo. Un contexto que demanda fórmulas renovadas de gobernanza internacional más democráticas, abiertas e inclusivas que favorezcan la generación de consensos internacionales para la provisión de bienes públicos globales, pero, que a su vez combata las resistencias de ciertos actores internacionales.

» El cumplimiento de esta nueva agenda internacional se desarrolla en un marco geopolítico internacional que, actualmente, se encuentra entre aquellos que abogan por la imprescindible coordinación multilateral y aquellos que defienden la unilateralidad y la recuperación de la soberanía nacional. De ahí que, el trabajo de las redes transnacionales pueda fortalecer los vínculos entre sociedades y favorecer la transición hacia un modelo de gobernanza internacional más equilibrado y horizontal.

» Este nuevo modelo de desarrollo solo será posible con la participación activa de todos los actores relevantes: poderes públicos (ejecutivos, legislativos, organismos internacionales), academia, sector privado, organizaciones internacionales, sociedad civil y ciudadanía. Esto pasa por la generación de nuevos modelos de alianzas público-privadas capaces de gestar soluciones escalables y que maximicen la aportación de cada uno de los actores.

» De entre las lecciones aprendidas de la Agenda 2030, cabe destacar que, muchos de los resultados esperados no se cumplieron porque el papel de los Gobiernos locales, actores imprescindibles para el desarrollo, fue instrumental. Reconoce que, sin el concurso activo de los poderes locales, desde la formulación de los objetivos hasta la evaluación de su impacto, no se logrará su consecución.

Así, partiendo del último reto señalado, la Agenda 2030 ha situado a los territorios como pieza clave de una óptima implementación de los objetivos o, dicho de otra forma, pone el énfasis en lo que actualmente se denomina: la localización de los ODS. Atendiendo a esta aproximación, se entiende por localización el proceso que tiene en cuenta los contextos subnacionales en el logro de la Agenda 2030, desde el establecimiento de los objetivos y metas, hasta la determinación de los medios de implementación, y el uso de indicadores para medir y monitorear el progreso hecho. La localización guarda relación sobre cómo los Gobiernos locales y regionales pueden dar apoyo para alcanzar los ODS a través de la acción desde abajo y cómo, a su vez, los ODS pueden ofrecer un marco para la política de desarrollo local (GTF, 2016). La localización se trata por tanto de un proceso de adaptación de la agenda global a las características y circunstancias de cada territorio, siendo consustancial con la necesidad de participación de los diferentes actores que componen una comunidad (CIDOB, 2015). Sin embargo, la localización de los ODS requiere de una serie de premisas básicas que deben ser incorporadas a la agenda política que consisten en (FEMP, 2018a):

» Constituir un marco de referencia y de principios inspiradores que pueden ofrecer un enfoque global a iniciativas sectoriales y locales ya existentes.

» Evitar aproximaciones burocráticas y ofrecer una perspectiva movilizadora y de liderazgo local. Se trata de una oportunidad de movilización pública, social y privada para acometer los retos fundamentales de las ciudades.

» Desarrollar instrumentos para mejorar la transparencia, el buen gobierno y la participación ciudadana, como claves para fomentar una gobernanza abierta que permita un desarrollo sostenible compartido y cocreado con la ciudadanía.

\section{La Agenda 2030 pone el énfasis en lo que actualmente se denomina: la localización de los ODS}


» Sensibilizar a la población e incorporar a los diferentes actores relevantes, públicos y privados, tanto en el diseño de la planificación como en la ejecución de las actuaciones para su efectiva implementación.

» Fortalecer las capacidades de los Gobiernos locales, incluyendo el desarrollo de herramientas participativas, innovadoras y sostenibles, que garanticen la apropiación por parte de la ciudadanía.

» Apostar por un liderazgo transversal y legitimado en el seno de las administraciones territoriales, capaz de movilizar a los departamentos sectoriales y de hacerlo desde una perspectiva integral.

» Articular mecanismos de gobernanza multinivel en el territorio como requisito necesario para concitar el diálogo y la coordinación entre los diferentes actores, tanto en términos estratégicos como en términos de movilización de los recursos políticos, sociales y económicos.

» Establecer sistemas de seguimiento de progresos y de rendición de cuentas, ofreciendo un marco armonizado de recogida de información que fortalezca las capacidades de las administraciones locales para un tratamiento ágil de la información. Los ODS son, también, una revolución en términos de gestión de datos.

» Promover la acción del Gobierno local en el ámbito de acción exterior y de cooperación para conectar sus actuaciones en el ámbito internacional a través de las redes y organismos habilitados para ello.

\section{El papel de los Gobiernos locales en la Agenda 2030: ODS 11 y nueva Agenda Urbana}

La Agenda 2030 de Desarrollo Sostenible, aprobada por la Asamblea General de las Naciones Unidas el 25 de septiembre de 2015, como se ha señalado, se ha convertido en la agenda internacional más ambiciosa de la humanidad. Además de su carácter universal, que implica a todos los países, por primera vez la Agenda se asume desde una perspectiva holística: social, cultural, económica y medioambiental, donde la promoción de alianzas estratégicas con aquellos que abogan por los mismos objetivos se convierte en esencial y necesaria. Asimismo, "la aprobación de la Nueva Agenda Urbana (NAU) en la Tercera Conferencia de las Naciones Unidas sobre Vivienda y Desarrollo Sostenible - HABITAT III (2016) constituye una guía para orientar los esfuerzos en materia de desarrollo de las ciudades de cara a los próximos veinte años. La NAU llegó un año después de la Agenda 2030 creando un vínculo de refuerzo recíproco entre urbanización y desarrollo. La inclusión del ODS 11 para 'Hacer que las ciudades y los asentamientos humanos sean inclusivos, seguros, resilientes y sostenibles' es, en gran parte, el fruto de una intensa campaña de los gobiernos locales, sus asociaciones y la comunidad urbana” (FEMP, 2018b).

El ODS 11 representa un gran paso hacia adelante en el reconocimiento del poder transformador de la urbanización para el desarrollo, y en el papel de los líderes de las ciudades a la hora de impulsar el cambio global desde la base. Sin embargo, el papel de los Gobiernos locales en la consecución de la Agenda va más allá del objetivo 11, ya que todos los ODS tienen metas directa o indirectamente relacionadas con el trabajo diario de los Gobiernos locales y regionales. De ahí que, dichos niveles de gobierno sean responsables políticos, catalizadores del cambio y los mejor situados para vincular los objetivos globales con las comunidades locales. Prueba de
La Agenda 2030 de Desarrollo Sostenible se ha convertido en la agenda internacional más ambiciosa de la humanidad 
ello es el intenso periodo 2012-2016, donde los Gobiernos locales y regionales han sido muy activos alzando su voz y demostrando la importancia del trabajo en red en las negociaciones de todas las agendas globales. Esta importante labor de incidencia política ha tenido un impacto concreto en el reconocimiento de los Gobiernos locales en las mismas (FEMP, 2018c). Algunos ejemplos se pueden comprobar en la siguiente figura:

Figura 1. Referencias al ámbito local en las agendas y declaraciones internacionales

\section{Art. 42 de la declaración de Río+20 (2012)}

"Reafirmamos el papel fundamental que desempeñan los órganos legislativos y de gobierno a todos los niveles en la promoción del desarrollo sostenible. Reconocemos además la importancia de que todas las instancias pertinentes encargadas de adoptar decisiones participen en la planificación y aplicación de políticas de desarrollo sostenible"

\section{Art. 34 de la Agenda de Acción de Addis Abeba (2015)}

"Nos esforzaremos por prestar apoyo a las administraciones locales en sus esfuerzos por obtener ingresos, según proceda"

Art. 11.2 del Acuerdo de París sobre el Cambio Climático (2015)

"El fomento de las capacidades debería estar bajo el control de los países, basarse en las necesidades nacionales y responder a ellas, y fomentar la implicación de las Partes, en particular de las que son países en desarrollo, incluyendo los niveles nacionales, subnacionales y locales".

\section{Art. 45 de la Agenda 2030 (2015)}

“Reconocemos asimismo que los parlamentos nacionales desempeñarán un papel fundamental en el cumplimiento efectivo de nuestros compromisos promulgando legislación, aprobando presupuestos y garantizando la rendición de cuentas. Los Gobiernos y las instituciones públicas también colaborarán estrechamente en la implementación con las autoridades regionales y locales, las instituciones subregionales, las instituciones internacionales, la comunidad académica, las organizaciones filantrópicas, los grupos de voluntarios y otras instancias".

\section{Art. 8 de la Nueva Agenda Urbana - Hábitat III (2016)}

"Reconocemos las contribuciones de los Gobiernos Nacionales, así como las contribuciones de los Gobiernos subnacionales y locales, a la definición de la Nueva Agenda Urbana y tomamos nota de la Segunda Asamblea Mundial de Autoridades Locales y Regionales".

\section{Art. 86 del Nuevo Consenso Europeo de Desarrollo (2017)}

“El logro de la mayoría de los ODS depende en gran medida de la participación activa de las autoridades locales y regionales. La UE y sus Estados miembros apoyarán reformas de transparencia, rendición de cuentas y descentralización, cuando corresponda, para empoderar a las autoridades regionales y locales para una mejor gobernanza y un mejor impacto en el desarrollo, y para abordar mejor las desigualdades dentro de los países. Apoyarán los procesos para ayudar a las personas a interactuar efectivamente con el gobierno local en todas las etapas de la planificación e implementación de políticas, y fortalecerán su cooperación con las autoridades locales y otras subnacionales, incluso a través de la cooperación descentralizada".

Fuente: Aportaciones de la FEMP al Plan de Acción Nacional de la Agenda 2030 (FEMP, 2018c)

Como puede comprobarse en la figura 1, los Gobiernos locales, regionales y las asociaciones que los representan llevan incidiendo desde hace más de una década en diferentes acuerdos internacionales, esto ha permitido participar activamente en el diseño de la nueva Agenda 2030 y de la NAU. Ambas agendas internacionales reconocen la importancia que ostentan los Gobiernos locales para garantizar una implementación efectiva de dichos objetivos. Dicho reconocimiento es una prueba más de los logros alcanzados por la organización mundial Ciudades y Gobiernos Locales Unidos (CGLU) por medio del Grupo de Trabajo Global de Gobiernos Locales y Regionales ${ }^{1}$. 
Las recomendaciones de Naciones Unidas transitan del reconocimiento a la aplicación a través de diez acciones principales que se detallan a continuación (PNUD, 2016):

Figura 2. Recomendaciones de naciones Unidas para la implementación de la Agenda 2030

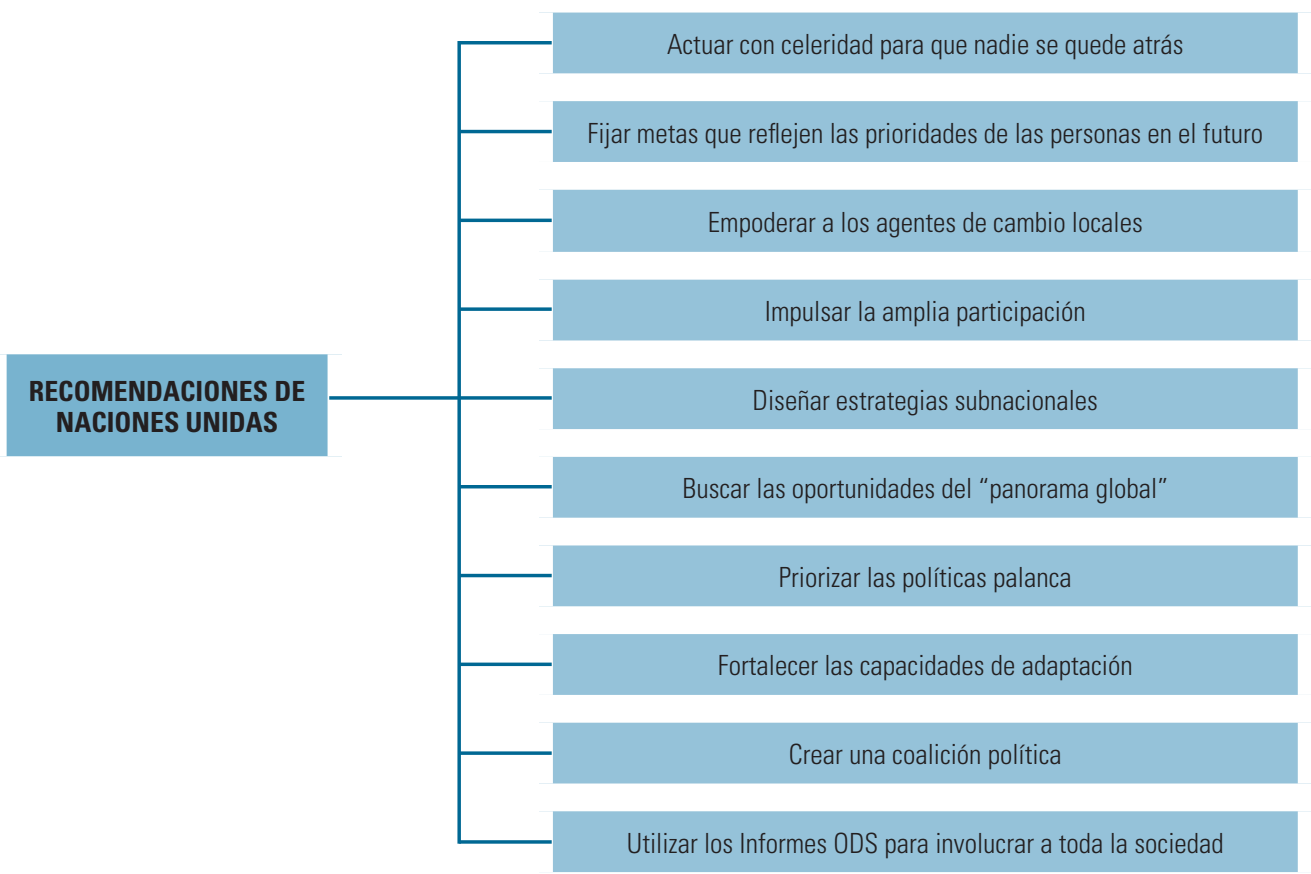

Fuente: Informe Desde los ODM hasta el desarrollo sostenible para todos: Lecciones aprendidas tras 15 años de práctica (PNUD, 2016)

Los logros alcanzados por el Grupo de Trabajo Global en relación al reconocimiento de los Gobiernos locales tanto en la Agenda 2030 como en la Nueva Agenda Urbana, se han convertido en bases sólidas y estables para la construcción de las estrategias de acción tanto desde una perspectiva sectorial como territorial. La agenda global de los Gobiernos locales y regionales se sintetiza en el Compromiso de Bogotá y las recomendaciones que este contiene basadas en el IV Informe Global sobre Democracia Local y Descentralización que consiste en una acción con triple perspectiva: (1) la acción global, que aborda tres potentes factores para contribuir al desarrollo local y nacional: la gobernanza global, la financiación internacional y la cooperación descentralizada; (2) La acción nacional, centrada en la necesidad de impulsar las reformas legales, institucionales y políticas pertinentes a nivel nacional; y (3) la acción local, que aborda cómo los Gobiernos subnacionales pueden contribuir a la consecución de los ODS, del Acuerdo de París, del Acuerdo Marco de Sendai y de la Nueva Agenda Urbana (CGLU, 2016). Algunos ejemplos ayudan a visualizar la importancia de la acción de los Gobiernos locales en la efectiva implementación de la Agenda 2030 (Alonso, Huitrón, \& Santander, 2017):

» La lucha contra la pobreza y la mejora en los niveles de provisión y calidad de los servicios sociales básicos al conjunto de la población: gran parte de esos servicios son provistos desde las administraciones descentralizadas. 
» La construcción de sociedades incluyentes, a través del combate a la desigualdad: son los poderes regionales y locales los que tienen un papel clave en la identificación y corrección de los procesos de exclusión y marginación social en que se asientan esas desigualdades.

» La generación de sociedades pacíficas y bien gobernadas, erradicando la inseguridad y la violencia: es importante que ese proceso se construya desde la base territorial más cercana a los ciudadanos, para facilitar de ese modo el enraizamiento de los mecanismos de participación y control democrático por parte de la ciudadanía.

» Asegurar las condiciones de sostenibilidad ambiental de los procesos de desarrollo, mejorando la gestión de los recursos naturales y la protección de los ecosistemas más frágiles: los poderes locales y regionales son clave para acometer esas políticas, tratando de hacer compatible la sostenibilidad con el progreso económico y social de las comunidades afectadas.

» Crear tejido productivo y alentar una senda de crecimiento económico, con capacidad de generar empleo de calidad: el papel que los poderes regionales y locales tienen en esos procesos es fundamental, pues pueden generar actividad económica sobre bases territoriales bien definidas, a través de alianzas entre actores, que incluyan a la pequeña y mediana empresa local.

En suma, el papel de los Gobiernos locales y regionales y sus asociaciones en el logro de los ODS es crucial, ya que es en dicho nivel donde se puede preservar el enfoque de igualdad para su implementación a nivel nacional, ya que todos incluyen metas relacionadas con competencias y responsabilidades de la esfera local, principalmente en la prestación de servicios básicos y en la promoción de un desarrollo territorial endógeno, inclusivo y sostenible (FEMP, 2018a).

\section{Iberoamérica una apuesta por los ODS: de lo global a lo local}

\subsection{La comunidad iberoamericana frente a los ODS}

Los Estados miembros de la Conferencia Iberoamericana han otorgado especial prioridad en sus políticas públicas a la implementación de la Agenda 2030. En el camino hacia la XXVI Cumbre Iberoamericana de La Antigua (Guatemala) que se celebra bajo el lema "Una Iberoamérica próspera, inclusiva y sostenible”, han confluido dos prioridades: recoger insumos para formular las propuestas que se aprueben sobre la temática del desarrollo sostenible y dirigir su acción a la lógica de los ODS a la que se están orientando todos los actores de desarrollo (Alonso, Huitrón, \& Santander, 2017). El estudio llevado a cabo por la Secretaria General Iberoamericana (SEGIB) "Iberoamérica y los Objetivos de Desarrollo Sostenible" constituye un punto de partida esencial, un primer diagnóstico en la región en relación a la nueva agenda de desarrollo de referencia imprescindible.

En este sentido, es relevante señalar el punto de partida de dicho estudio cuando indica que: "los cambios en el sistema internacional y las nuevas demandas de gobernanza imponen una adecuación del rol de la comunidad iberoamericana ante la nueva agenda de desarrollo. En este sentido, tres características de la cooperación iberoamericana parecen especialmente adecuadas para dicha adaptación” (Alonso, Huitrón, \& Santander 2017): en primer lugar, se parte de un nuevo escenario donde el desarrollo y la cooperación que la Agenda 2030 formula se fundamenta en relaciones horizontales que superan las clásicas dicotomías: desarrollado-subdesarro-

\section{El papel de los} Gobiemos locales y regionales y sus asociaciones en el logro de los ODS es crucial, ya que es en dicho nivel donde se puede preservar el enfoque de igualdad para su implementación a nivel nacional 
llado, rico-pobre o norte-sur. La cooperación iberoamericana se ha caracterizado en la última década por el tipo de cooperación que promueven los ODS. En segundo lugar, el espacio iberoamericano ha sido un marco de cooperación multinivel y multiactor desde su inicio. Son incontables las iniciativas en la región en la que participan diferentes niveles de gobierno - local, regional, nacional e internacional-y múltiples actores públicos y privados — universidades, sector privado, sociedad civil, partidos políticos, etc.- - El arraigo de este tipo de cooperación, la experiencia y la red de actores lo convierte en un escenario propicio para dar un paso más en relación a la gobernanza de la Agenda. En tercer lugar, la cooperación iberoamericana se ha destacado por el desarrollo de su acción tanto en el nivel político como en el técnico, originando espacios de incidencia, de fortalecimiento institucional y de definición de políticas públicas en ámbitos muy diversos, tales como la lucha contra la pobreza, la violencia de género, la corrupción, el cambio climático, entre otros, que hoy son ejes centrales de la Agenda 2030 (Alonso, Huitrón, \& Santander, 2017).

Sin embargo, a esos rasgos cabe sumar algunos factores post 2015 que hoy posicionan a la región en una situación singular: en primer lugar, un año después de la aprobación de la Agenda 2030, tuvo lugar la Conferencia HABITAT III en Quito (Ecuador) que aprobó la Nueva Agenda Urbana. El eje de discusión y movilización entorno a la Agenda 2030 y la NAU por parte de Gobiernos locales de la región iberoamericana ha supuesto, sin duda, una apropiación de mayor calado que en otras regiones del mundo, incluida la Unión Europea. En segundo lugar, la movilización de recursos y la generación de alianzas se encuentran en la lógica de la región debido a la comprensión de esa doble naturaleza de donante-receptor, de la apuesta por la cooperación sur-sur y de la integración de organismos internacionales en la apuesta nación-región-mundo. América Latina y el Caribe está constituido por 42 países, de los cuales 25 son de renta media y tiene la mayor proporción de países de ingresos medios en todo el mundo (22,94\% del total), seguido de Asia Oriental y el Pacífico $(21,10 \%)$ y Europa y Asia Central (20,18\%) (SEGIB, 2018). En tercer lugar, la Agenda 2030 dota de una narrativa común a nivel mundo, que tiene un relato propio en Iberoamérica que ha sido favorecido por la SEGIB. La cumbre de La Antigua debe ser el espacio que consolide ese marco de políticas, que permita pasar del compromiso a la acción. En cuarto lugar, la puesta en marcha de planes, estrategias, mecanismos de articulación e iniciativas a nivel nacional se han convertido en referentes de transmisión más allá de la propia región. En quinto lugar, el espacio iberoamericano es líder en materia de intercambio de experiencias, fortalecimiento de capacidades y construcción de metodologías de trabajo conjuntas en ámbitos de interés mutuo. Esta cooperación es especialmente relevante y reseñable entre los niveles locales (ciudades, provincias, regiones).

En contraposición a esos rasgos característicos y factores, se constatan algunas valoraciones y percepciones de carácter preventivo en torno a la incidencia de la Agenda 2030 en la región en sus primeras fases (Alonso, Huitrón, \& Santander, 2017): en primer lugar, se pone el acento en las disparidades existentes entre los países iberoamericanos. Ello supone un reto de cara a establecer una hoja de ruta armonizada por lo que debe prestarse especial atención al ámbito institucional y de la gobernabilidad democrática en la región; en segundo lugar, los planes de acción a nivel nacional deben contemplar medidas adecuadas al contexto país, deben ser realistas y medibles. Existe, por tanto, una preocupación entorno al escaso grado de conocimiento y manejo de los indicadores en los que esta agenda se concreta; en tercer lugar, existen importantes retos para el adecuado despliegue práctico de la agenda, que conecta directamente con la generalizada percepción de que no existen medios y capacidades estadísticas suficientes en los

El gje de discusión y movilización entorno a la Agenda 2030 y la nAU por parte de Gobiernos locales de la región iberoamericana ha supuesto una apropiación de mayor calado que en otras regiones del mundo, incluida la Unión

\section{Europea}


países iberoamericanos para dar adecuado seguimiento a los ODS; finalmente, destaca el difuso y desigual compromiso político con la agenda y su insuficiente incorporación a los marcos de trabajo de las diversas áreas de gobierno.

Con todo, desde la aprobación de la Agenda 2030 el dinamismo en su incorporación ha sido especialmente relevante con respecto a otras regiones del mundo. Tal y como concluye el estudio Iberoamérica y los Objetivos de Desarrollo Sostenible la comunidad iberoamericana se revela como un espacio de trabajo conjunto que puede ostentar "un papel fundamental para proyectar la voz, visión e intereses de un conjunto de países cuya identidad compartida se vería de otra forma mucho más difuminada — y resultaría más difícilmente reconocible- en el escenario internacional. En este marco, y dentro de las amplias demandas de gobernanza que, como se ha visto, se han abierto en el sistema internacional en los últimos años, la comunidad iberoamericana presenta unas especificidades cuya adecuada capitalización puede resultar de clara relevancia para los retos que comporta la nueva agenda de desarrollo y el logro de los ODS" (Alonso, Huitrón, \& Santander, 2017).

La oportunidad de la Cumbre Iberoamericana de Jefes y Jefas de Estado y de Gobierno en La Antigua (Guatemala) se enmarca en un contexto internacional marcado por la nueva agenda internacional. Los veintidós países miembros están llamados a definir un plan de acción conjunto. La conferencia iberoamericana, con más de veinticinco años de historia de trabajo e impulso de alianzas entre diversos actores que fortalecen Iberoamérica, hoy cuenta con un espacio institucionalizado y consolidado que permite dar carta de naturaleza a una de las principales exigencias de la Agenda 2030, contar con el ámbito de Gobierno local y establecer mecanismos de gobernanza multinivel. Los Gobiernos locales iberoamericanos a través del foro que les reúne cada dos años se convierten en aliados y socios imprescindibles para desarrollar los mandatos de las cumbres y analizar el camino que se debe seguir en el futuro.

\subsection{Enfoque local de la Agenda 2030 en Iberoamérica: gobernanza multinivel y gobierno abierto}

Actualmente más de la mitad de la población del mundo vive en ciudades. Esa relación es incluso mayor en América Latina, que ha sido tradicionalmente una región altamente urbanizada. Las ciudades proporcionan la prestación de los servicios básicos a la población, impulsan una fluida interacción entre las personas y generan dinamismo en el ámbito cultural, social y económico. Pero los territorios con un elevado grado de urbanización también dan lugar a diferentes tipos de desequilibrios: inadecuadas infraestructuras, creación de asentamientos irregulares, problemas medioambientales, instituciones ineficaces, entre otros. La Agenda 2030 quiso atender estos aspectos a través del objetivo 11 y las ciudades iberoamericanas fueron pieza clave en los insumos que finalmente se incorporaron en la NAU (Alonso, Huitrón, \& Santander, 2017).

Los Gobiernos locales y las ciudades que forman parte de la región iberoamericana se enfrentan a desafíos comunes que, afectando directamente a sus ciudadanías, a menudo transcienden sus fronteras territoriales, sus competencias administrativas y sus capacidades económicas. Algunos de ellos son: el cambio climático y la creciente vulnerabilidad de las poblaciones a sus efectos, la pérdida de biodiversidad, la contaminación atmosférica, el agotamiento y la sobreexplotación de los recursos naturales que afectan de manera evidente y amenazan las posibilidades de las próximas generaciones; el crecimiento de la desigualdad y la profundización de las condiciones de pobreza que golpean las condiciones de vida y limitan el potencial de desarrollo

\section{La conferencia} iberoamericana, con más de veinticinco años de historia de trabajo e impulso de alianzas entre diversos actores que fortalecen Iberoamérica, hoy cuenta con un espacio institucionalizado y consolidado 
de poblaciones y comunidades; la existencia de diferentes tipos de violencias que se expresan con dureza minando la libertad y la autonomía de las personas; y la recuperación de la confianza en la administración local, previniendo y combatiendo la corrupción, garantizando la provisión eficiente, abierta, competitiva y justa de los servicios públicos, generando mejores canales de comunicación que permitan acercar el Gobierno a la ciudadanía (FIGL, 2018).

Por todo ello, los Gobiernos locales iberoamericanos expresaron su firme compromiso con la Agenda 2030 en la Declaración de Quito en 2016 Ciudades sostenibles y Asentamientos Urbanos para Todos en el marco de la conferencia HABITAT III. El rol de los Gobiernos locales, durante los debates y la redacción de dichos acuerdos fue relevante e imprescindible, logrando incorporar en la agenda global la importancia de poner el foco en "el territorio", en la necesidad de fortalecer mecanismos de gobernanza abierta, transparencia y participación ciudadana y en el abordaje de una nueva articulación multinivel con los Gobiernos nacionales, regionales y la comunidad internacional para fortalecer el diálogo con el resto de los actores clave en el desarrollo y la cohesión territorial (FIGL, 2018). Así, lo reconoce la SEGIB cuando afirma que "la eficaz adopción de la Agenda, necesita de una adecuada división del trabajo y una fluida comunicación entre los diversos niveles de gobierno, en el seno de un país. Esto es especialmente relevante en la relación entre el Gobierno de la nación y los poderes locales y regionales. Ello implica que buena parte de los ODS solo podrán ser plenamente realizados si se logra implicar a los poderes subnacionales en la tarea y se opta por una localización o territorialización de los mismos" (Alonso, Huitrón, \& Santander, 2017).

En este sentido, resulta sumamente relevante la posición que ostenta Iberoamérica con respecto a otras regiones del mundo. La cooperación iberoamericana, a través de la SEGIB, ha consolidado un espacio para el diálogo y la articulación de políticas que cuenta con la perspectiva nacional, regional y local de la región. La garantía de participación de todos los actores institucionales, y muy particularmente del ámbito local, supone un reconocimiento del rol clave de las ciudades y de las redes que les asocian, para poder abordar de manera eficaz buena parte de los retos que la nueva agenda plantea. De ahí que, la cooperación iberoamericana articule y apoye los esfuerzos que están realizando las instituciones iberoamericanas de representación local para generar conocimiento y coordinación sobre el enfoque territorial de la Agenda 2030, y a su vez, invite a formular todas las propuestas que contribuyan a fortalecer este ejercicio de adaptación a la conferencia iberoamericana. Una visión multinivel de la región que permite integrar la perspectiva de lo local y regional en las cumbres de jefes y jefas de Estado a través del instrumento legitimado para ello: el Foro Iberoamericano de Gobiernos Locales.

\subsubsection{La gobernanza multinivel iberoamericana: el Foro Iberoamericano de Gobiernos Locales}

El Foro Iberoamericano de Gobiernos Locales (FIGL) surgió en el año 2006 con el objetivo de ser un espacio permanente de encuentro, debate y cooperación entre municipios y provincias, de los países que integran la Comunidad Iberoamericana de Naciones, y la voluntad de ser reconocido como marco legítimo por parte de la Cumbre Iberoamericana de Jefes de Estado y de Gobierno. Desde ese momento hasta la actualidad, ha evolucionado hasta convertirse en un lugar de encuentro de los municipios, las regiones y las asociaciones iberoamericanas. De ahí que se celebre con anterioridad a la reunión de la cumbre para poder contribuir con ideas, iniciativa o proyectos y participar así activamente en la construcción de la agenda iberoamericana.

\section{Esta visión}

multinivel de la región permite integrar la perspectiva de lo local y regional en las cumbres de jefes y jefas de Estado a través del instrumento legitimado para ello: el Foro Iberoamericano de Gobiernos

\section{Locales}


Se constituye como un espacio para la creación de vínculos en los distintos niveles del espacio iberoamericano y particularmente con la Secretaría General Iberoamericana. En síntesis, un marco común de trabajo, de apoyo y complemento, desde la óptica local y regional, mediante recomendaciones y compromisos concretos, a los resultados de las cumbres (FIGL, 2018).

En noviembre de 2007, durante la XVII Cumbre Iberoamericana de Jefes de Estado y de Gobierno (Santiago de Chile), se institucionalizó formalmente el Foro Iberoamericano de Gobiernos Locales como parte del sistema de la conferencia iberoamericana. La Secretaría Pro Tempore de Chile optó por incluir el foro entre los encuentros que formarían parte, ese año, de la Conferencia Iberoamericana y, en vista de ello, la SEGIB propuso formalmente la incorporación del Foro en el Sistema Iberoamericano. El foro se lleva a cabo antes de la Cumbre de Jefes de Estado y de Gobierno, a fin de que sus conclusiones puedan ser dadas a conocer y tomadas en cuenta por los mandatarios iberoamericanos. El foro está vinculado con los temas que se traten en el marco de la conferencia, sin que ello signifique desconocer su dinámica propia y las preocupaciones e intereses de sus miembros.

En el siguiente cuadro puede comprobarse la celebración de los distintos foros, sus temáticas y principales resultados:

Figura 3. Síntesis celebración Foro lberoamericano de Gobiernos Locales (2006-2016)

\begin{tabular}{|c|c|c|c|c|}
\hline AÑo & SEDE & FECHA & TEMÁTICA /TÍTULO & RESULTADOS \\
\hline 2006 & $\begin{array}{l}\text { MONTEVIDEO } \\
\text { (URUGUAY) }\end{array}$ & Octubre & $\begin{array}{l}\text { Migraciones: Desde lo } \\
\text { global a lo local }\end{array}$ & Declaración de Montevideo \\
\hline 2007 & $\begin{array}{l}\text { VALPARAÍSO } \\
\text { (CHILE) }\end{array}$ & Octubre & $\begin{array}{l}\text { Gobiernos Locales como } \\
\text { articuladores de la cohesión } \\
\text { social }\end{array}$ & Declaración de Valparaíso \\
\hline 2008 & $\begin{array}{l}\text { SAN SALVADOR } \\
\text { (EL SALVADOR) }\end{array}$ & $\begin{array}{l}4 / 5 \\
\text { septiembre }\end{array}$ & $\begin{array}{l}\text { Juventud y Desarrollo: } \\
\text { Políticas públicas locales }\end{array}$ & $\begin{array}{l}\text { Borrador de la Carta Iberoamericana de la } \\
\text { Autonomía Local } \\
\text { Borrador reglamento de participación y } \\
\text { funcionamiento del foro } \\
\text { Declaración de San Salvador }\end{array}$ \\
\hline 2009 & $\begin{array}{l}\text { LISBOA } \\
\text { (PORTUGAL) }\end{array}$ & $\begin{array}{l}\text { 19/20 } \\
\text { noviembre }\end{array}$ & $\begin{array}{l}\text { Innovación municipal, una } \\
\text { salida para la crisis }\end{array}$ & $\begin{array}{l}\text { Declaración de Lisboa } \\
\text { Carta iberoamericana de autonomía local }\end{array}$ \\
\hline 2010 & $\begin{array}{l}\text { MAR DEL PLATA } \\
\text { (ARGENTINA) }\end{array}$ & 15/16 octubre & $\begin{array}{l}\text { Educación para la inclusión } \\
\text { social }\end{array}$ & Declaración de Mar del Plata \\
\hline 2011 & $\begin{array}{l}\text { ASUNCIÓN } \\
\text { (PARAGUAY) }\end{array}$ & Septiembre & $\begin{array}{l}\text { Gobernabilidad local, } \\
\text { Descentralización y reforma } \\
\text { del Estado }\end{array}$ & $\begin{array}{l}\text { Reglamento de participación y } \\
\text { funcionamiento del foro } \\
\text { Declaración de Asunción }\end{array}$ \\
\hline 2012 & MADRID & Octubre & $\begin{array}{l}\text { Políticas locales de } \\
\text { promoción empresarial y } \\
\text { creación de empleo }\end{array}$ & Declaración de Madrid \\
\hline 2013 & $\begin{array}{l}\text { CIUDAD DE } \\
\text { PANAMÁ (PANAMÁ) }\end{array}$ & Septiembre & $\begin{array}{l}\text { Los Gobiernos locales } \\
\text { iberoamericanos en el } \\
\text { nuevo contexto mundial }\end{array}$ & $\begin{array}{l}\text { Declaración de Panamá } \\
\text { Cumbres y foros bienales }\end{array}$ \\
\hline 2014 & $\begin{array}{l}\text { CIUDAD DE MÉXICO } \\
\text { (MÉXICO) }\end{array}$ & Noviembre & $\begin{array}{l}\text { Educación y Cultura para la } \\
\text { inclusión en el espacio local }\end{array}$ & $\begin{array}{l}\text { Declaración de Ciudad de México } \\
\text { Acuerdo de promover una instancia } \\
\text { ejecutiva de actuación permanente }\end{array}$ \\
\hline 2016 & QUITO (ECUADOR) & 16 de octubre & $\begin{array}{l}\text { El papel de los Gobiernos } \\
\text { locales iberoamericanos } \\
\text { en la construcción de la } \\
\text { Nueva Agenda Urbana y la } \\
\text { consecución de los ODS }\end{array}$ & $\begin{array}{l}\text { Declaración de Quito } \\
\text { Acuerdo sobre la creación de una } \\
\text { Secretaría permanente }\end{array}$ \\
\hline
\end{tabular}

Fuente: elaboración propia 
El reglamento del FIGL establece sus objetivos fundamentales: (1) establecer un ámbito de diálogo y de cooperación entre los Gobiernos locales para promover y fortalecer las relaciones entre los ciudadanos que viven en los territorios que representan y que constituyen, en el ámbito local, una red de profundas interrelaciones humanas de cohesión social que consolidan y dan vida a la Comunidad Iberoamericana de Naciones; (2) promover la solidaridad, la colaboración y la cooperación para el desarrollo entre las ciudades iberoamericanas, con el fin de enriquecer y profundizar las estrechas relaciones ya existentes; (3) fomentar acciones conjuntas en ámbitos culturales, sociales y políticos para fomentar un desarrollo sostenido y equilibrado de las ciudades iberoamericanas y una mejor convivencia entre los ciudadanos; (4) aportar a la conferencia iberoamericana la visión local en la discusión de los temas que concitan la atención de las autoridades estatales centrales que participan en los distintos ámbitos de la conferencia, con el objeto de que las propuestas emanadas del foro sean consideradas a la hora de definir políticas públicas y estrategias estatales.

Derivado de dichos objetivos, el FIGL se enfrenta a los mismos desafíos que el resto de la región con respecto a la implementación de la Agenda 2030, pero con una seña de identidad propia. Hoy los Gobiernos locales iberoamericanos están liderando procesos de localización e implementación de las nuevas agendas globales, pero resulta imprescindible fortalecer sus capacidades técnicas y políticas para que la acción local se constituya en un pilar fundamental en la consecución de las transformaciones propuestas. Avanzar en marcos de equidad, de cohesión, de participación ciudadana, de convivencia en paz y de sostenibilidad en todas las acepciones que promueven los diecisiete ODS es el trabajo que corresponderá definir a los máximos representantes de los municipios o provincias de cada país representados en el foro. El salto de la declaración a la acción, y por ende a la puesta en marcha de políticas correctoras de las vulnerabilidades, desigualdades y desequilibrios de la región, debe poner el foco en el qué y en el cómo. Por ello, se necesitan instituciones legítimas y capaces de desempeñarla en todas sus fases (formulación, implementación y evaluación). Una vez más los Gobiernos locales iberoamericanos están realizando un avance significativo en modelos de gobernanza abierta y colaborativa que está situando a la transparencia, el acceso a la información, la rendición de cuentas, la participación ciudadana y la prevención y lucha contra la corrupción en el centro de sus agendas locales, proporcionando espacio para el empoderamiento de las personas y colectivos, generando espacios de diálogo político con los actores sociales, auspiciando controles y mecanismos que garanticen la apropiación y fortaleciendo la confianza ciudadana en la gestión de los asuntos públicos (FIGL, 2018).

\subsubsection{Gobiernos locales abiertos: una palanca para la Agenda 2030 en Iberoamérica}

La profundidad, integralidad y multidimensionalidad que caracteriza a los ODS frente a agendas anteriores es uno de sus aspectos más positivos. Sin embargo, sobresalen específicamente algunos objetivos a los que se les atribuye una mayor relevancia o grado de prioridad en la región, entre ellos, el logro de la paz, la justicia y las instituciones sólidas, el ODS 16, que es considerado esencial para avanzar en materia de desarrollo en los países iberoamericanos (Alonso, Huitrón, \& Santander, 2017). Una de las insuficiencias que se imputa a los ODM es haber relegado las dimensiones no materiales del desarrollo, esto es, aquellas que tienen que ver con la defensa de los derechos humanos, el fomento de las condiciones adecuadas de gobernanza, la calidad de las instituciones y el impulso de la paz. Son todas, sin embargo, dimensiones decisivas del desarrollo, al menos desde una doble mirada: porque son dimensiones determinantes 
e intrínsecas del desarrollo, pero también porque son medios para alcanzar mejores resultados en la dirección del progreso. La Agenda 2030 se hizo eco de esa carencia e incorporó el ODS 16 para recoger estos aspectos, relacionados con los derechos, la paz y la gobernanza (Alonso, Huitrón, \& Santander, 2017). La transparencia, la participación y la rendición de cuentas, lo que hoy conocemos como gobierno abierto, se reconocen como eje básico de toda acción política y por tanto de la acción relativa a los ODS.

De ahí que la Agenda 2030 y los principios del gobierno abierto encuentren un espacio de complementariedad que otorga mayor visibilidad al papel de la transparencia, la participación ciudadana, las instituciones públicas responsables y la innovación tecnológica, como facilitadores del desarrollo sostenible para que prosperen sociedades inclusivas, justas y pacíficas. Fortalecer la complementariedad entre los elementos de gobernanza y gobierno abierto que engloban estas dos agendas será central para su exitosa implementación. Así, el ODS 16 se convierte en un vehículo para que los países avancen en la implementación de los ODS de dos formas: directamente, mediante el establecimiento de metas y objetivos centrados específicamente en la apertura, haciendo eco a sus propias metas, que buscan promover sociedades justas, pacíficas e inclusivas para todos a través de instituciones que rinden cuentas; e, indirectamente, al reconocer que los principios de gobierno abierto son fundamentales para el logro de una gama mucho más amplia de objetivos de desarrollo sostenible, al proporcionar herramientas para un mejor diseño de mecanismos responsables y transparentes que den seguimiento a la implementación de los ODS. Desde esta segunda perspectiva, el gobierno abierto puede convertirse en una política palanca, definida esta como aquellas políticas con capacidad de acelerar la implementación de los ODS, idóneas para romper silos o compartimentos sectoriales rígidos y atender a un desarrollo sostenible que conecte diferentes actores, sectores y políticas en una visión común integrada (Lopez Pagan, 2018; FEMP, 2018c; Gobierno de España, 2018).

Asimismo, tanto la Agenda 2030 como la NAU reconocen el papel que durante décadas han desarrollado los Gobiernos locales liderando experimentos de nuevas formas de toma de decisiones participativas de cara a lograr sociedades más sostenibles, efectivas y responsables para con los ciudadanos. Veinticuatro referencias a la participación ciudadana, veintidós referencias a la transparencia y a la rendición de cuentas, doce a procesos de colaboración y cocreación, y nueve a la utilización de las nuevas tecnologías, es el balance del reflejo de la inserción de las políticas de gobierno abierto en el marco de la NAU. Las políticas de gobierno abierto ofrecen un marco favorable para que los Gobiernos locales y regionales avancen en el logro de estos objetivos, ya que implican una nueva forma de gobernanza que favorece la transparencia y el acceso a la información pública, promueve la trazabilidad de las decisiones políticas, involucra a los ciudadanos en el ciclo de políticas públicas y fomenta el trabajo conjunto entre ciudadanos, administraciones y el sector privado (Lopez Pagan, 2018).

En el caso de Iberoamérica, la cooperación entre Gobiernos locales cuenta con décadas de experiencia, que ha dado sus frutos en un modelo único de cooperación caracterizado por conseguir resultados concretos que inciden en el fortalecimiento de las políticas públicas sectoriales, basado tanto en la cooperación técnica (intercambio de buenas prácticas, generación de redes, generación de diálogo político) así como en la creación de capacidades a través de la formación. En materia de políticas de transparencia, rendición de cuentas, participación ciudadana y gobierno abierto, Iberoamérica es una de las regiones donde más avances se están desarrollando. Ejemplo de ello, es la adopción de Carta Iberoamericana de Gobierno Abierto (CIGA), elaborada por el Centro Latinoamericano de Administración para el Desarrollo (CLAD) y aprobada

\section{La Agenda 2030} y la กAU reconocen el papel que han desarrollado los Gobiemos locales liderando experimentos de nuevas formas de toma de decisiones participativas 
en 2016 en XVII Conferencia Iberoamericana de Ministras y Ministros de Administración Pública y Reforma del Estado. La CIGA identifica y desarrolla una serie de criterios y principios orientadores para generar políticas públicas de gobierno abierto, que abarcan los tres pilares iniciales, a saber: transparencia, participación y colaboración; pero recogiendo otros aspectos necesarios como la rendición de cuentas, la innovación ciudadana, la calidad de los servicios públicos y la asociación Estado-sociedad como actores corresponsables en el desarrollo sostenible de los países. La CIGA expresamente reconoce el papel de los Gobiernos subnacionales y de los municipios abiertos "dada su proximidad, el rol que cumplen en el espacio territorial y la cercanía a las necesidades de la población, los Gobiernos subnacionales (regionales, departamentales) y locales (municipios, ayuntamientos) son una pieza fundamental en un modelo integral de gobierno abierto. En Iberoamérica son ya muchas las experiencias locales exitosas de apertura, que desde entornos metropolitanos como de ayuntamientos rurales y menos poblados, dan cuenta de que este nivel de proximidad con el ciudadano es clave en el desarrollo de iniciativas de gobierno abierto en la región" (CLAD, 2106).

La carta continúa expresando que por ello, se deberá buscar que los principios y mecanismos expresados "tengan una aplicación preferente y prioritaria en este nivel de Gobierno, no solo siendo considerados en los planes y estrategias nacionales, sino promoviendo acciones propias y marcos legales que permitan el desarrollo y consolidación de este modelo de gobernanza a nivel local, y que busque a su vez reducir la marginación, las desigualdades y permita la adecuada articulación del territorio, en donde cuestiones tales como la defensa y gestión de los recursos naturales, el desarrollo sustentable y la preservación del medio ambiente, jugarán un papel clave en la Agenda 2030" (CLAD, 2016).

Para avanzar en el cumplimiento de ambas agendas será imprescindible hacer un plan de acción en la región que promueva el gobierno abierto como política palanca en las estrategias país. Para ello resulta esencial: (1) revisar las dimensiones con las que el Banco Mundial mide la calidad institucional — voz y rendición de cuentas; estabilidad política; eficacia del gobierno; calidad de la regulación; Estado de derecho; y control de la corrupción-; (2) desarrollar las metas e indicadores establecidos para el ODS 16 que consisten en: reducir considerablemente la corrupción y el soborno en todas sus formas; crear a todos los niveles instituciones eficaces y transparentes que rindan cuentas; y garantizar la adopción en todos los niveles de decisiones inclusivas, participativas y representativas que respondan a las necesidades; e (3) incorporar un análisis cualitativo sobre los procesos llevados a cabo en los diferentes niveles de gobierno de la región en relación a las políticas de gobierno abierto.

\section{Conclusiones}

Iberoamérica constituye un espacio de integración política que ha renovado su discurso y su marco de acción en torno a la Agenda de Desarrollo Sostenible. En dicho escenario de actuación conjunta, es imprescindible priorizar y declinar metas sobre las que los países de la región puedan progresar de una forma armonizada. La propia riqueza y heterogeneidad de la comunidad iberoamericana puede dar lugar a procesos de implementación de la agenda que se enmarquen en diferentes velocidades. Pero ello, debe capitalizarse en positivo. En un marco de cooperación y solidaridad entre los Estados iberoamericanos, deben encontrarse y fijarse las fórmulas pertinentes para el apoyo mutuo y el intercambio de experiencias, donde aquellos que por su contexto puedan progresar más ayuden a aquellos que encuentran más dificultades. 
Buscar un lenguaje compartido, construir un hilo conductor que derive en una narrativa singular de Iberoamérica para el cumplimiento de los ODS cuenta con un presupuesto de partida común: los responsables públicos enfrentan el reto de reconectarse con su ciudadanía de una manera renovada y de fortalecer su confianza en la gestión pública. Asimismo, la región está posicionada como líder en la formulación de nuevas formas de gobernanza abierta que pueden dar respuesta a dicho reto. La ciudadanía, cada vez más activa y concienciada, exige más información sobre la acción de su Gobierno, así como una mayor transparencia y responsabilidad, un mejor acceso a la información y nuevos mecanismos de diálogo con las administraciones públicas a todos los niveles. Esta conexión es esencial para la construcción de territorios más incluyentes, responsables, participativos y eficientes en la prestación de servicios públicos. Para responder a estas demandas, muchos poderes públicos de ámbito local, regional y central del espacio iberoamericano han adoptado el gobierno abierto en su agenda administrativa y política, como un nuevo modelo de gobernanza que brinda una oportunidad para las administraciones públicas de todos los niveles. Iberoamérica, desde el compromiso con la Agenda 2030 cuenta con una herramienta esencial, su conocimiento y apuesta por el gobierno abierto. Iberoamérica debe aprovechar esa experiencia y ponerla al servicio del desarrollo sostenible de su comunidad.

\section{Bibliografía}

Alonso, J. A., Huitrón, A., \& Santander, G. (2017). Iberoamérica y los Objetivos de Desarrollo Sostenible. Instituto Complutense de Estudios Internacionales (ICEI-UCM). Madrid: Secretaria General Iberoamericana (SEGIB). Disponible en https://www.segib.org/wpcontent/uploads/LosODSeIberoam--ricaweb.pdf

CGLU. (2016). IV informe del Observatorio Mundial sobre Democracia Local y Descentralización: Cocreando el futuro urbano (GOLD IV). Disponible en https://www.uclg.org/sites/ default/files/gold_esp-web.pdf

CIDOB. (2015). La localización de los ODS. Disponible en https://www.cidob.org/es/publicaciones/documentacion/dossiers/dossier_ods_2015_2030/objetivos_de_desarrollo_sostenible_la_agenda_2030_del_compromiso_a_la_practica/la_localizacion_de_los_ods

CLAD. (2016). Carta Iberoamericana de Gobierno Abierto, Centro Latinoamericano de Administración para el Desarrollo, adoptada por la XXV Cumbre Iberoamericana de Jefes de Estado y de Gobierno. Cartagena de Indias, Colombia, 28 y 29 de octubre de 2016. Disponible en https://www.clad.org/images/declaraciones/CIGA-Octubre-2016.pdf

FEMP. (2018a). Think Europe: Compromiso 2030. Notas conceptuales del encuentro celebrado en el marco del Consejo de Municipios y Regiones de Europa los días 16 a 18 de enero de 2018. Soria. Disponible en www.localizandoods.es

FEMP. (2018b). Estrategia de la FEMP para la implementación de la Agenda 2030 de los Objetivos de Desarrollo Sostenible, 26 de junio 2018. Madrid. Disponible en www. localizandoods.es

FEMP. (2018c). Aportaciones al Borrador 0 del Plan de Acción para la implementación de la Agenda 2030, mayo 2018. Madrid. Disponible en: www.localizandoods.com

FIGL. (2018). Declaración del XI Foro Iberoamericano de Gobiernos Locales, adoptada el 7 de noviembre de 2018 en Madrid. 
GTF, UNDP, \& UN Habitat. (2016). Roadmap for localizing the SDGs: implementation and monitoring at subnational level. Disponible en https:/www.uclg.org/sites/default/files/ roadmap_for_localizing_the_sdgs_0.pdf

Gobierno de España. (2018). Plan de Acción para la implementación de la Agenda 2030: Hacia una Estrategia Española de Desarrollo Sostenible, 29 de Junio. Madrid. Disponible en http://www.exteriores.gob.es/Portal/es/SalaDePrensa/Multimedia/Publicaciones/Documents/PLAN\%20DE\%20ACCION\%20PARA\%20LA\%20IMPLEMENTACION\%20 DE\%20LA\%20AGENDA\%202030.pdf

López Pagán, J. (2018). La ventana de oportunidad del gobierno abierto en España: un análisis desde el ámbito local. Granada: CEMCI.

ONU. (2015). Transformar nuestro mundo: la Agenda 2030 para el Desarrollo Sostenible, Resolución aprobada por la Asamblea General el 25 de septiembre de 2015. Agenda 2030 para el Desarrollo Sostenible. Disponible en https://unctad.org/meetings/es/SessionalDocuments/ares70d1_es.pdf

ONU. (2016). Nueva Agenda Urbana. Disponible en http://habitat3.org/wp-content/uploads/ NUA-Spanish.pdf

PNUD. (2016). Desde los ODM hasta el desarrollo sostenible para todos: Lecciones aprendidas tras 15 años de práctica. Disponible en http:/www.undp.org/content/dam/undp/ library/SDGs/Spanish/ES_f_UNDP_MDGs-to-SDGs_web.pdf

SEGIB. (2018). Nota conceptual. Una Iberoamérica próspera, inclusiva y sostenible. XXVI Cumbre de Jefes de Estado y de Gobierno de Iberoamérica La Antigua Guatemala 2018. Disponible en http://cumbreiberoamericana2018.gt/Uploads/Documents/201810051136040_ Nota_conceptual_Guatemala.pdf 